\title{
PRECISION OF EPHEMERIDES FOR SPACE MISSIONS
}

\author{
B. G. MARSDEN \\ Smithsonian Astrophysical Observatory
}

One of the most extensive programs of systematic observations of minor planets currently being conducted is that at the Crimean Astrophysical Observatory. The observed positions, together with the residuals from the predictions in the annual Ephemeris volumes, have been listed in the Minor Planet Circulars for several years now; and inspection of the residuals yields information about the accuracy of minor planet ephemerides as a whole.

The observations are made using a $40 \mathrm{~cm} \mathrm{f/4} \mathrm{double} \mathrm{astrograph,} \mathrm{the} \mathrm{limiting}$ magnitude being about 18. Between June 1968 and October 1969, corresponding to one synodic period of an average minor planet, about 40 percent of all the numbered objects were observed. These observations may be regarded as reasonably representative of all the planets. Observations were made of 60 of the objects with numbers in the 100's and only 29 of those in the 1100 's, but in general the distribution was surprisingly uniform.

Figure 1 shows, on a logarithmic scale, the distribution of the residuals in right ascension for the 694 planets involved. We have not considered the

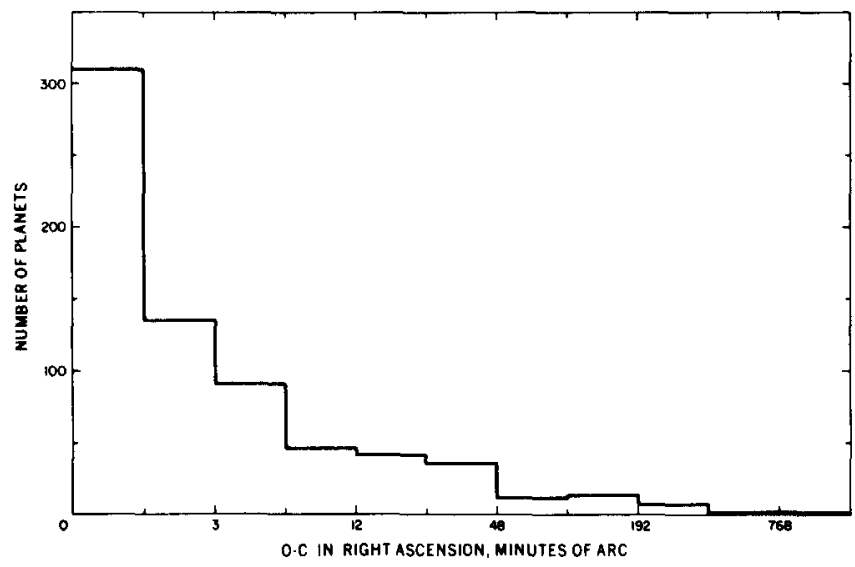

Figure 1.-Distribution of the $O-C$ residuals in right ascension for 694 minor planets observed between June 1968 and October 1969 at the Crimean Astrophysical Observatory. 
residuals in declination, but they are generally not as important. It may be seen that 64 percent of the planets had residuals of less than $3^{\prime}$; and, again, there was little difference between high and low numbered objects. For astrometric purposes this limit may be regarded as very satisfactory. If one wishes to send a space probe to a minor planet, however, it certainly is not satisfactory. At a typical opposition distance of $1.8 \mathrm{AU}$ from Earth, $3^{\prime}$ corresponds to $2.3 \times 10^{6} \mathrm{~km}$. Furthermore, 16 percent of the minor planets had residuals exceeding $12^{\prime}$, and 1.6 percent (11 objects in all) were found $3^{\circ}$ or more from their predicted positions. Four of these 11 objects, having numbers in the 1200 to 1500 range, had been observed relatively poorly in the past, and the new Crimean observations have been highly valuable for redetermining their orbits. This was not true of the lower numbered objects; but, again, for three of these (two of which were brighter than $14 \mathrm{mag}$ ), the current Ephemeris volume contains new orbits. It should be pointed out perhaps that some of the large residuals are due to misidentifications, although this effect should not greatly distort the general features of the distribution.

In general, however, thanks to the Cincinnati Observatory, the Institute of Theoretical Astronomy in Leningrad, the Latvian State University Observatory, and the modern computers available to these institutions, the immense task of keeping track of the minor planets is well under control. In the 1971 volume, there are only four minor planets for which the ephemerides have been calculated without consideration of perturbations, and two of these have not been identified with certainty since their discovery oppositions. For the majority of minor planets (those calculated in Leningrad) perturbations by Jupiter and Saturn are applied; although for about 30 percent of them, allowance is made for perturbations by all the planets from Venus to Neptune.

This is, of course, the point: If perturbations by all the major planets are consistently applied, and if new differential corrections are performed every 10 $\mathrm{yr}$ or so, it should be possible to supply, for 90 to 95 percent of the numbered planets, ephemerides that are in error by no more than a few seconds of arc. For astrometric purposes, it would obviously be pointless to make the necessary effort, although observers making photometric and polarimetric measurements would find it highly convenient if the planets that concern them could be relied upon to be within, say, $1^{\prime}$ of their predicted positions. Perhaps the time has come to draw up a comprehensive selected list, comprising 200 or 300 objects, even very faint ones, of potential astrophysical interest and for which reliable ephemerides would be guaranteed.

For space mission purposes, one is interested only in a handful of very selective objects. The observed positions would be reduced to the FK4 system, the orbit corrections performed as rigorously as possible, and perturbations by all the major planets taken into account. Perhaps the positions and masses of these perturbing planets will have to be improved. It is rather ironic that so many attempts have been made in recent years to use observations of minor planets for determining the mass of Jupiter because the average of these new determinations is practically identical with the IAU adopted value. On the 
other hand, there are at least five minor planets-433 Eros, 887 Alinda, 944 Hidalgo, 1221 Amor, and 1566 Icarus-for which recent orbit calculations have easily revealed severe inadequacies in the adopted masses of Earth or Saturn. Many of the bright, well-observed minor planets have geocentric distances in the range 0.9 to $1.2 \mathrm{AU}$ at favorable oppositions. Very great care in the orbital computations on 4 Vesta and 8 Flora, for example, could produce predictions a few years ahead that are good to 1 ". This corresponds to an uncertainty of 700 to $900 \mathrm{~km}$ in the plane of the orbit. The real difficulty is that we have no direct information about the range, although it might be reasonable to multiply these uncertainties by a factor between, say, 2 and 5 .

On the other hand, a more convenient and interesting objective for a space probe may be one of the minor planets that approach Earth very closely. Two obvious choices are Icarus and Eros. Although the best predictions for the former were in error by $20^{\prime \prime}$ at the time of recovery in 1968, this corresponds to a distance, in the orbit plane, of only $1100 \mathrm{~km}$. The extensive series of observations obtained that year should improve matters in the future (although to my knowledge no observations have been made since 1968, and not for the lack of suitable occasions); and, furthermore, the radar experiments established the range very exactly. Eros has a much longer observational history; and its larger size, lower orbital eccentricity, and larger perihelion distance make it a more attractive target. Neither of these objects has an aphelion distance of more than $2.0 \mathrm{AU}$, however; and they can hardly be regarded as representative minor planets. It is also questionable whether what may be our only chance of subjecting a minor planet to close inspection from space should be spent on a chunk of iron, which is what Eros is quite widely thought to be.

We suggest, therefore, that 887 Alinda be put forward as another candidate. Though far from a typical minor planet, its aphelion distance is almost $4 \mathrm{AU}$, and it spends most of its time in and around the main belt. That it is one of the half dozen or so minor planets most likely to be defunct cometary nuclei could allow us to settle the very important question of interrelation of minor planets and comets. Alinda's libration about the 3:1 mean motion resonance with Jupiter implies that it is approximately in a 1:4 mean motion resonance with Earth, and it has been observed only on its close approaches to Earth. Following its discovery in 1918, it was observed faithfully every 4 yr until 1942; it was not observed again until late 1957, when two positions were measured on a single night. Two $\mathrm{yr}$ ago, in preparation for the favorable 1969-70 opposition, we made a redetermination of the orbit. Although recognizing the great weight we were placing on the isolated pair of observations in 1957, we concluded that it was necessary to use the mass of Earth consistent with the radar determinations of the astronomical unit, rather than the IAU value. The maximum residual of this orbit in $1969-70$ was about $3 "$, even though Alinda came within $0.23 \mathrm{AU}$ of Earth; and as a result of a solution that incorporated the new observations, these residuals were reduced by a maximum of 2". 6 . For no orbital element did the correction exceed three times the mean error of its determination. A change of 2".6 at $0.23 \mathrm{AU}$ 
corresponds to $430 \mathrm{~km}$, whereas the formal spatial difference between the two orbit solutions was $540 \mathrm{~km}$.

In view of the paucity of observations since 1942 , this result may seem very encouraging; but it should be pointed out that the orbit has been exclusively conditioned to observations covering an arc of about 7 months centered on perihelion. We have no information about the error in the remainder of the orbit; however, because any space probe would presumably intercept Alinda near perihelion, this is of little consequence. As for the more severe difficulty of establishing the range, it is worth considering the possibility of bouncing a radar beam off Alinda on its next return. At the end of 1973 it will approach to within $0.14 \mathrm{AU}$, or 3.5 times the least distance of Icarus in 1968. Although cataloged as 1.5 mag brighter (absolutely) than Icarus, the recent observations suggest that $3 \mathrm{mag}$, or a factor of 16, may be more correct. Alinda could thus be expected to have a radius of $3 \mathrm{~km}$, and the strength of the radar echo will be $3.5^{4} / 16$, nine times weaker than that for Icarus. Success, coupled with concentrated astrometric and physical observations in 1973-74, and possibly even astrometric observations at other oppositions, ${ }^{1}$ could then lead the way to a prediction in error by not more than a few tens of kilometers and a space probe launched to Alinda in 1977.

\footnotetext{
${ }^{1}$ Very faint images of Alinda were photographed by E. Roemer in May and June 1971 with the Steward Observatory's $229 \mathrm{~cm}$ reflector on Kitt Peak. This tiny minor planet was then more than $3.6 \mathrm{AU}$ from the Sun.
} 